\title{
Counter incision is a safe and effective method for alternative reservoir placement during inflatable penile prosthesis surgery
}

\author{
Dominic Grimberg ${ }^{1}$, Sabrina Wang ${ }^{2}$, Evan Carlos $^{1}$, Brent Nosé $^{1}$, Shelby Harper ${ }^{2}$, Aaron C. Lentz ${ }^{1}$ \\ ${ }^{1}$ Division of Urology, Duke University Medical Center, Durham, NC, USA; ${ }^{2}$ Duke University School of Medicine, Durham NC, USA \\ Contributions: (I) Conception and Design: D Grimberg, E Carlos, AC Lentz; (II) Administrative support: D Grimberg, B Nosé, S Wang, S Harper; \\ (III) Provision of study materials or patients: AC Lentz; (IV) Collection and assembly of data: D Grimberg, B Nosé, S Wang, S Harper, E Carlos; (V) \\ Data analysis and interpretation: D Grimberg, E Carlos, B Nosé, AC Lentz; (VI) Manuscript writing: All authors; (VII) Final approval of manuscript: \\ All authors. \\ Correspondence to: Dr. Dominic Grimberg. Division of Urology, Duke University Medical Center, Room 1573, White Zone, Durham, NC, 27710, \\ USA. Email: dominic.grimberg@duke.edu.
}

Background: Alternative reservoir placement is increasingly popular during inflatable penile prosthesis (IPP) surgery to prevent intraperitoneal positioning, bowel, bladder, or vascular injury in patients with prior pelvic surgeries. Counter incision (CI) can be used for submuscular reservoir placement in high risk patients, however series exploring the safety remain limited.

Methods: A database of IPP surgeries was queried for use of a CI during reservoir placement to compare 90-day clinical outcomes in a retrospective case-control study. Primary outcome was device infections, with secondary outcomes including reservoir herniation, hematoma, device malfunction rates, and operative times. Groups were compared using Kruskal-Wallis and Chi-Squared tests, with multivariate logistic regression models to identify predictors of infectious complications.

Results: A total of 534 cases met criteria, of which $51(9.6 \%)$ used a CI for reservoir placement. The CI cohort included significantly more removal and replacements, $45.1 \%$ vs. $20.9 \%(\mathrm{P}<0.001)$. Thirty-one CI patients $(61.0 \%)$ had undergone prior prostatectomy compared to $134(27.7 \%)$ non-CI patients $(\mathrm{P}=0.001)$. The most common reasons for CI were prior prostatectomy and inguinal hernia repair. Median operative time was 17 minutes longer in the $\mathrm{CI}$ group (74 vs. 57 minutes, $\mathrm{P}<0.001$ ). Device infection rates were similar ( $2.0 \%$ vs. $4.1 \%, \mathrm{P}=0.71)$, as were rates of hematoma (5.9\% vs. $2.7 \%, \mathrm{P}=0.19)$, and device malfunction $(0.0 \%$ vs. $1.4 \% \mathrm{P}=1.00)$.

Conclusions: Complication rates were similar between CI and non-CI cohorts, even in a subset where approximately half the cases were removal and replacements. For physicians not comfortable with alternative placement through a penoscrotal or infrapubic incision, this offers a reasonable alternative and permits use of three-piece devices in patients with a hostile pelvis.

Keywords: Alternative reservoir placement; counter incision (CI); device infection; erectile dysfunction; penile prosthesis

Submitted May 17, 2020. Accepted for publication Sep 11, 2020.

doi: 10.21037/tau-20-923

View this article at: http://dx.doi.org/10.21037/tau-20-923

\footnotetext{
$\wedge$ ORCID: 0000-0003-2289-4781.
} 


\section{Introduction}

Penile prosthesis placement is central to treatment of erectile dysfunction unresponsive to more conservative therapies. Despite advances in nerve sparing techniques in the robotic prostatectomy era, rates of post-prostatectomy erectile dysfunction remain high and a large fraction of these patients would benefit from inflatable penile prosthesis (IPP) placement (1). Unfortunately, due to postprostatectomy scarring of the prevesical space and effective "peritonealization" of the bladder, traditional three-piece IPP reservoir placement blindly into the space of Retzius via the external inguinal ring has been linked to severe complications given the close proximity of bowel, bladder, and major vascular structures (2-6). Cadaver studies have revealed the external inguinal ring is only $2.5-4 \mathrm{~cm}$ from the external iliac vein and 2-4 cm from a non-decompressed bladder in a patient with normal pelvic anatomy (7). Specific patient populations are at even higher risk for such complications, including those with prior surgeries that alter pelvic anatomy such as radical prostatectomy and inguinal hernia repair $(8,9)$.

Due to the risk of severe complications associated with traditional reservoir placement in high risk patients, many prosthetic surgeons have opted to avoid the retropubic space and instead place reservoirs in alternative locations (5,10-13). Among the alternative locations for reservoir placement, the most frequently used are submuscular or high submuscular (HSM) positions, posterior to the rectus muscle but anterior to the transversalis fascia which can be achieved through a penoscrotal incision via the external inguinal ring $(5,13)$. By avoiding the space of Retzius, a submuscular position is advantageous by eliminating the risk of intraperitoneal placement and associated bowel, bladder, or vascular complications $(14,15)$. However, these are blind placements and the accuracy of the technique in achieving the expected positioning has been questioned by cadaveric studies (16). Furthermore, dilation of the external inguinal ring may increase the risk of reservoir herniation and need for subsequent revision, reported at up to $1.34 \%$ (17).

For patients with altered surgical planes from pelvic surgeries, especially radical prostatectomy, use of an inguinal counter incision (CI) for submuscular reservoir placement has been proposed to minimize complication risk $(8,9)$. This technique likely also avoids the increased reservoir herniation rate reported with submuscular placement via the external inguinal ring, and potentially reduces risk of injury to the epigastric vessels compared to blind submuscular placement $(8,17)$. A study of 31 patients with a history of pelvic surgeries undergoing a CI and lateral retroperitoneal reservoir placement reported no infections or malfunctions at 2 years (8). Survey data from a recent urology trainee cadaver lab reported significant participant anxiety with blind reservoir placement into the space of Retzius given the proximity to vital structures (18). Many urologists who are not high-volume implanters share this concern, especially in high risk patients, and we believe should consider a CI for alternative reservoir placements in those patients. We have anecdotally noted an increase in referrals for reservoir complications from occasional implanters not familiar with alternative reservoir placement, and in response, wanted to ensure our institution's trainees had a safe, reproducible option for IPP reservoir placement in a hostile pelvis.

Based on small studies, CIs are now an accepted technique for reservoir placement in patients with complex or altered pelvic anatomy, however a large-scale granular outcomes assessment is lacking. In this single institution retrospective study, we aim to report our experience using a CI for submuscular reservoir placement in patients at risk of complication with traditional reservoir placement due to prior pelvic surgeries. We present the following article in accordance with the STROBE reporting checklist (available at http://dx.doi.org/10.21037/tau-20-923).

\section{Methods}

We retrospectively reviewed a single institution database of patients who underwent penile prosthesis surgery between July 2013 and 2019 to compare outcomes between surgeries with and without CIs. These represent a single, reconstructive urology fellowship trained surgeon's series at a tertiary referral center with an emphasis on complex IPP surgeries. Inclusion criteria included age greater than or equal to 18 years, insertion of an IPP, and complete data including at minimum 90 days of follow up. The study was conducted in accordance with the Declaration of Helsinki (as revised in 2013). The study was approved by the institutional review board of Duke University (Pro00101391) and because of the retrospective nature of this research the requirement for informed consent was waived.

\section{Surgical technique}

Surgical and perioperative care was standardized for all 
patients regardless of CI status. Preoperatively all patients used a Hibiclens scrub the night before surgery, underwent hair removal, and were given pre-operative antibiotics in accordance with contemporary AUA guidelines-vancomycin, gentamycin, and cefazolin for most cases. There was no difference in perioperative antibiotics between single incision and CI patients. A 10-minute betadine scrub followed by chlorhexidine and alcohol-based prep was used for surgical prep. The vast majority of patients underwent placement via a penoscrotal approach, with an infrapubic approach used in a small minority. We adopted a "no-touch" technique as described by Eid starting in the spring of 2019 (19). CIs were made primarily in the left lower quadrant and dissection carried out until the rectus fascia was reached. The fascia was then opened, a submuscular space was created deep to the rectus muscle, and a nasal speculum was utilized to aid reservoir placemen. Low profile reservoirs (Conceal or Cloverleaf) were used in all CI cases. Following reservoir placement and tunneling of the tubing to the penoscrotal incision, the fascia overlying the reservoir was reapproximated and skin closed. Obese patients were positioned in Trendelenburg to allow the pannus to fall away from the operative field. In anticipation for potential future renal transplantation, select end stage renal disease (ESRD) patients underwent a lower midline CI with submuscular reservoir placement to avoid the transplant Gibson incision. In general, in patients with a normal contralateral inguinal canal and pelvis, we make an initial attempt at removing the reservoir from the penoscrotal incision. If not removed easily, or is deep in the pelvis, it is drained and decommissioned. In patients with a significant pelvic surgery history (robotic prostatectomy plus inguinal hernia, cystectomy, abdominal perineal resection, low anterior resection, femoral arterial bypass) we typically used a CI for reservoir removal. Blind reservoir removal through a penoscrotal incision can be very treacherous due to nearby vascular structures and potential for reservoir migration. CIs used in removal and replacement cases were made over the patient's existing reservoir, with the new reservoir placed into the old reservoir space after thorough irrigation with Zosyn, vancomycin, and amphotericin antibiotic solution and bacitracin irrigation (20). A 10-French Blake drain was placed in the scrotum intraoperatively following closure of bilateral corpora. A compressive dressing was placed at the end of surgery and the prosthesis was left in the erect position. Postoperatively, all patients remained in the hospital for observation overnight. The dressing and drain were removed on the morning of post-operative day one at which point the device was deflated.

\section{Statistical analysis}

The database was queried for basic demographic, surgical, and clinical data, and the cohort stratified by use of a CI for reservoir placement at the time of surgery. The primary outcome was comparing device infection rates, defined as infections requiring explantation, between patients in whom a CI was used for reservoir placement to those without a CI. Secondary outcomes included comparison of operative time and additional complications such as hematoma, reservoir herniation, device malfunction, and CI wound complications (hematoma, deep or superficial infection, and/ or dehiscence). Complications occurring within 90 days of surgery were recorded. Finally, a descriptive analysis of the indications for CI was performed. Patients without 90-day complication rates were excluded from analysis, thereby using a case deletion strategy to manage missing data.

De-identified data were analyzed with R 3.6.1 (Vienna, Austria) using R Studio 1.2.1 with the "tidyverse", "dplyr", "janitor", and "tableone" packages installed. KruskalWallis and Chi-Squared tests were used for continuous and categorical variables respectively. Thereafter, separate multivariable logistic regression models were run to determine predictors for any complication and device infection. Covariates used included age, smoking status, body mass index (BMI), diabetes, removal and replacement, use of a CI, and operative time. These covariate predictors were selected as historic predictors of infectious complications.

\section{Results}

There were a total 589 penile prosthesis surgeries over the study period and after excluding those with missing outcome data $(n=16)$ and malleable prostheses $(n=39)$, we identified 534 which met our inclusion criteria with 51 $(9.5 \%)$ using a CI for reservoir placement. Patients had a mean follow up of 7.6 months and all had a minimum of 90-day follow up. Our cohort of CI patients was demographically similar to the remainder of the population (Table 1), with a slightly lower BMI (27.0 vs. 29.91, $\mathrm{P}=0.002)$ and rate of diabetes $(25.5 \%$ vs. $41.0 \%, \mathrm{P}=0.045)$, and as expected a higher median total number of implant surgeries (2.0 vs. 1.0, $\mathrm{P}=0.004)$. Surgical details were also comparable (Table 2), aside from the fact that $44.2 \%$ of CI cases were implant removal and replacements compared to only 
Table 1 Cohort demographics

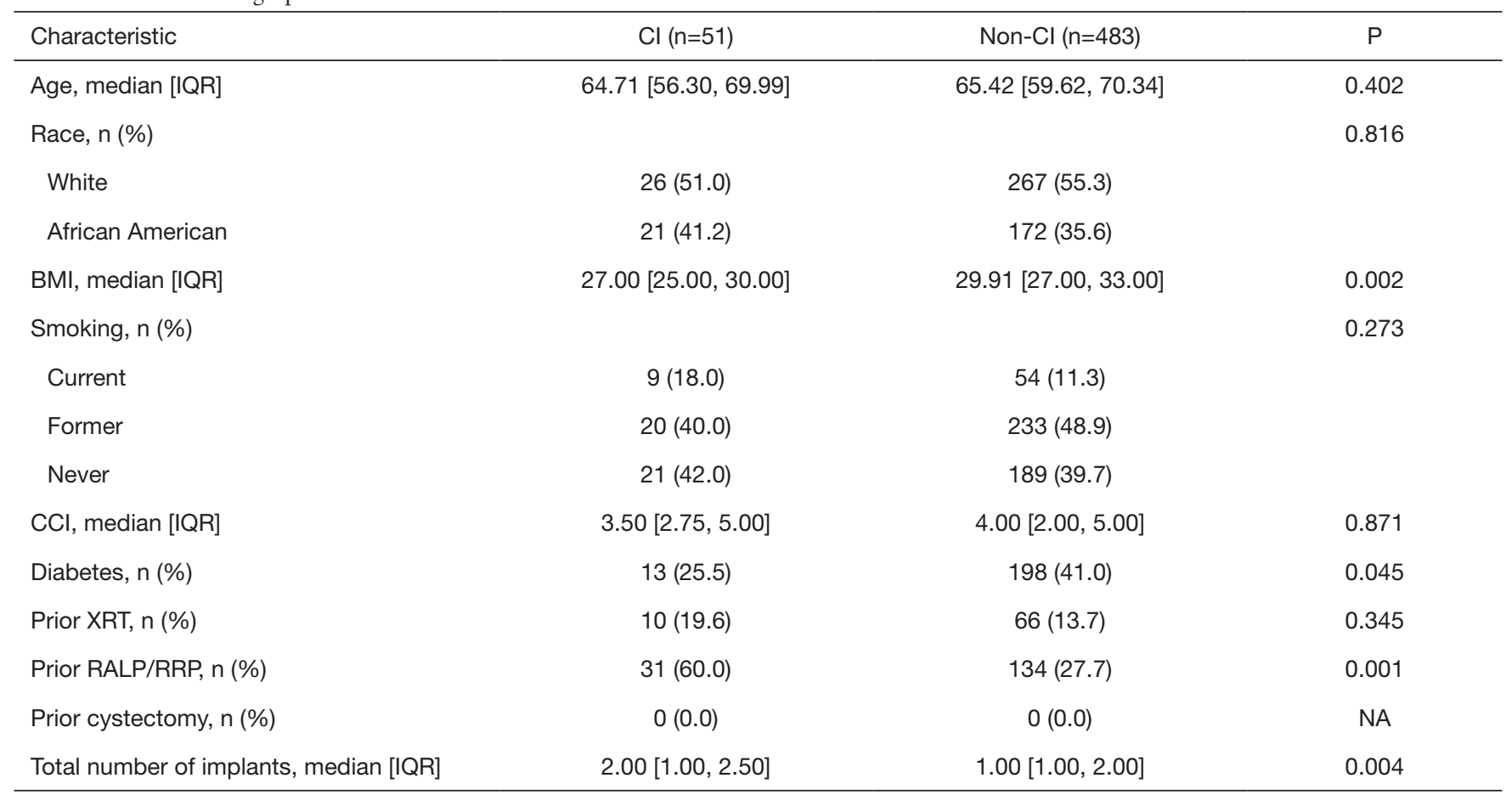

$\mathrm{Cl}$, counter incision; BMI, body mass index; CCI, Charlson comorbidity index; XRT, radiotherapy; RALP, robotic assisted laparoscopic prostatectomy; RRP, retropubic radical prostatectomy.

Table 2 Surgical details

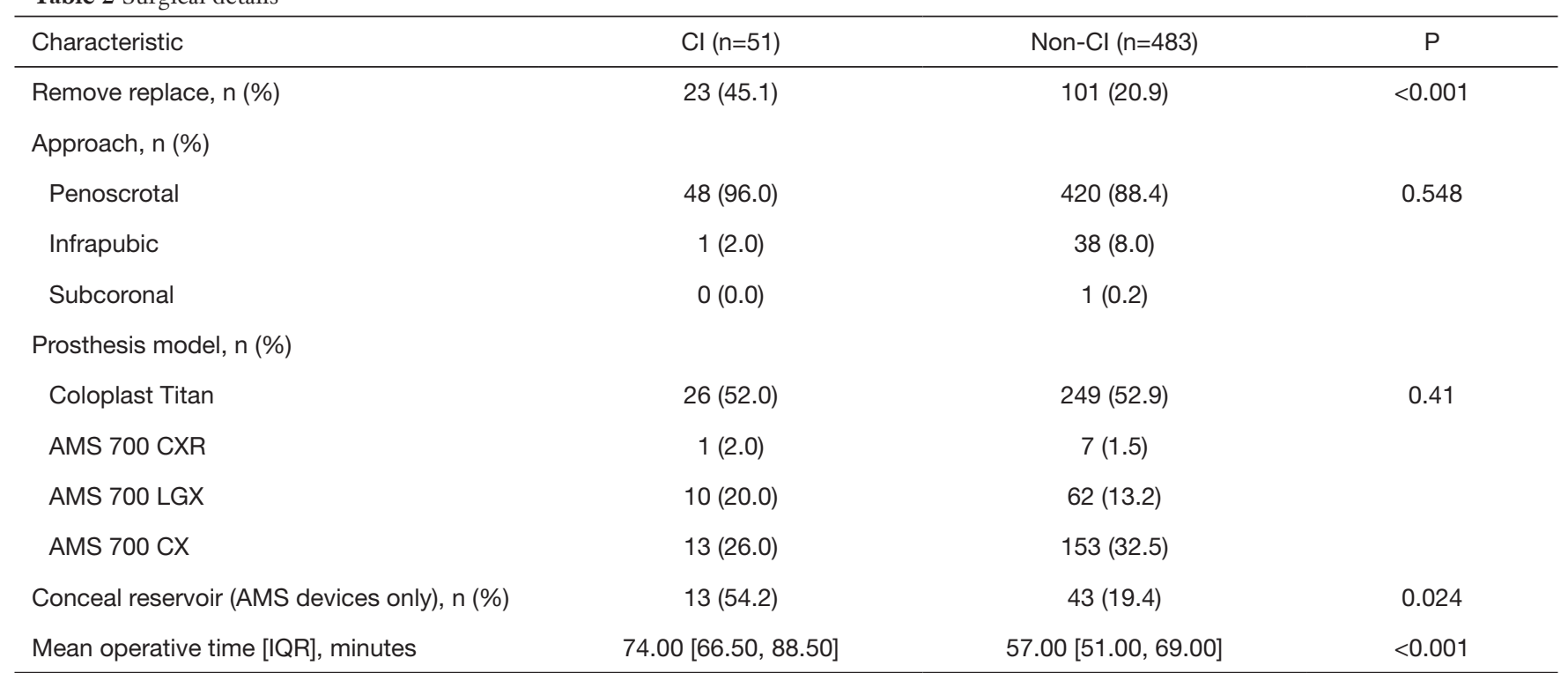

$\mathrm{Cl}$, counter incision. 


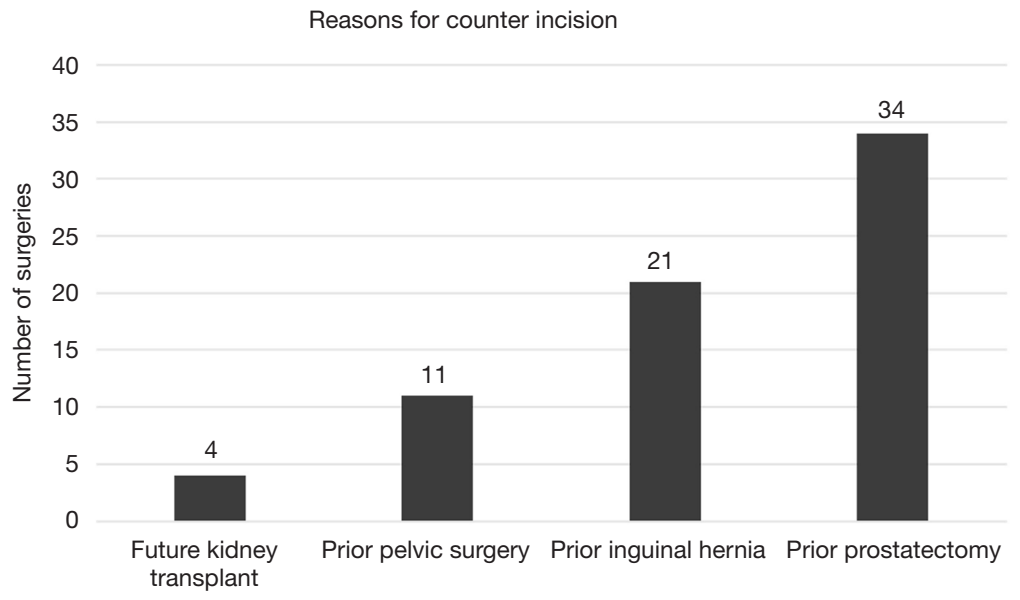

Figure 1 Histogram of patients' reasons for CI. CI, counter incision.

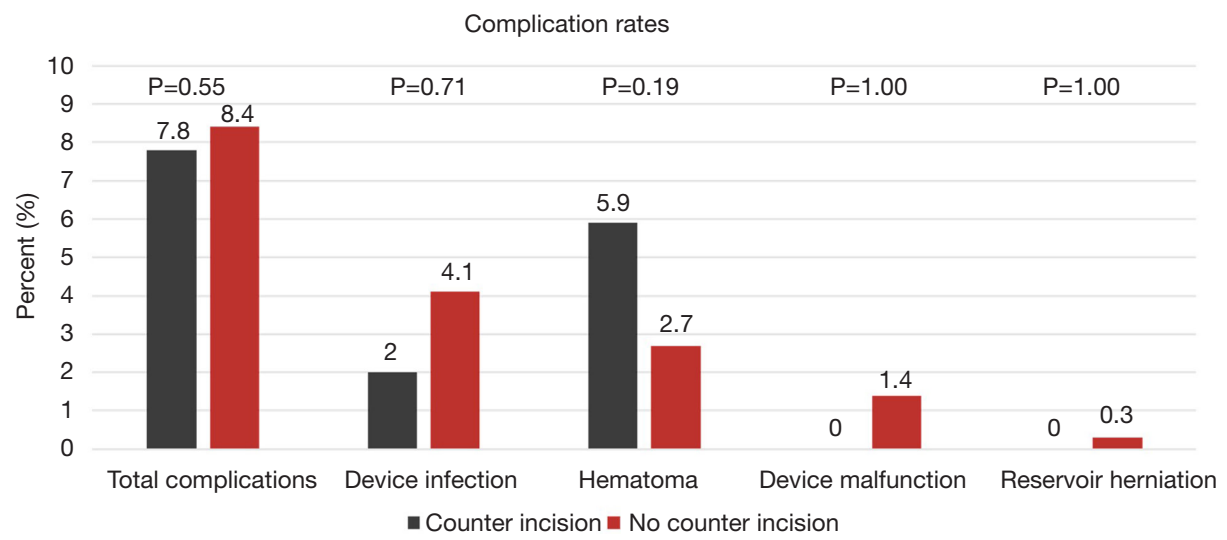

Figure 2 Complication comparisons between CI $(n=51)$ and non-CI cohort $(n=483)$ by univariate analyses. CI, counter incision.

$22.7 \%$ in the non-CI group $(\mathrm{P}=0.001)$. There was only one infrapubic approach $(2 \%)$ that used a CI, compared to $38(8 \%)$ in the non-CI cohort. This was in patient who had undergone a Mulcahy salvage procedure through an infrapubic incision 4 months prior. His surgical history was also notable for prior inguinal hernia repair, laparoscopic sigmoidectomy, vasectomy, and open cholecystectomy. The malleable prosthesis was removed and inflatable cylinders inserted through the infrapubic incision, and due to the patient's extensive past surgical history a CI was used for left lower quadrant submuscular reservoir placement. Cases using a CI had a higher median operative time by 17 minutes compared to those that did not (74 vs. 57 minutes, $\mathrm{P}<0.001$ ).

Ninety-eight CI indications were reported for the 51 surgeries (Figure 1). Most common reasons were prior prostatectomy (open or robotic/laparoscopic, $n=34$ ) and inguinal hernia repair (open or robotic/laparoscopic, $n=21$ ). A CI was also often used for removal and replacement cases $(\mathrm{n}=28)$, frequently in combination with another indication such as prior prostatectomy $(n=7)$. Finally, an additional four ESRD patients had a CI for HSM midline reservoir placement to avoid the Gibson incision of a future renal transplant. Of note, all four were first time implantees with no prior pelvic surgeries or contraindications to traditional placement.

By univariate analysis, the complication profile was similar between surgeries using a CI and those that did not (Figure 2). Total complication rates were $7.8 \%$ (4/51) for CI compared to $8.4 \%$ (41/483) for non-CI surgeries $(\mathrm{P}=0.546)$. One device infection was noted in the CI cohort $(2.0 \%)$ compared to $20(4.1 \%)$ in the non-CI cohort $(\mathrm{P}=0.71)$. 
Table 3 Multivariate logistic regression of predictors for any complication, device infection, and cellulitis

\begin{tabular}{|c|c|c|c|c|}
\hline Covariate & \multicolumn{2}{|c|}{ Any complication } & \multicolumn{2}{|c|}{ Device infection } \\
\hline Age & $0.974[0.948,1.006]$ & 0.112 & $0.966[0.924,1.020]$ & 0.151 \\
\hline \multicolumn{5}{|l|}{ Smoking } \\
\hline Never & 1.0 (referent) & 0.872 & 1.0 (referent) & \\
\hline Current & $0.833[0.220,2.541]$ & & $3.061[0.318,27.933]$ & 0.295 \\
\hline Diabetic & $1.220[0.577,2.556]$ & 0.597 & $1.088[0.259,4.359]$ & 0.904 \\
\hline BMI & $1.016[0.969,1.059]$ & 0.431 & 1.019 [0.937. 1.071] & 0.525 \\
\hline Remove and replace & $1.432[0.468,4.110]$ & 0.514 & $0.649[0.060,4.439]$ & 0.685 \\
\hline
\end{tabular}

$\mathrm{BMI}$, body mass index; $\mathrm{Cl}$, counter incision.

Only one complication of the CI site was observed-a single instance of superficial cellulitis which resolved with a short course of oral antibiotics and did not lead to device infection. After correcting for potential confounders using the multivariate models, use of a CI was not associated with total complications [OR: $1.57(0.50-4.30) ; \mathrm{P}=0.410$ ] or device infection [OR: $1.01(0.99-1.03) ; \mathrm{P}=0.810$ ] (Table 3).

We observed two reservoir-related complications in the non-CI cohort both in removal and replacement cases, including an intravesical reservoir placement and one delayed reservoir herniation requiring re-operation. The intravesical reservoir placement occurred in a removal and replacement case for malfunctioning prosthesis placed 13 years prior. The old reservoir was drained and decommissioned, and a new reservoir placed in the contralateral space of Retzius. Flexible cystoscopy at the completion of the case demonstrated an intravesical placement of the new reservoir, which was then removed, but the cylinders and pump were left in situ. After foley catheter drainage for 1 month and a negative cystogram, the patient underwent submuscular placement of a new reservoir via CI 4 months later which was connected to his cylinders and has since recovered without issue. In the CI cohort, one patient with a history of robotic prostatectomy and subsequent salvage radiation experienced an intraoperative iliac artery and vein injury during a removal and replacement for device malfunction. This was during the initial dissection of his existing reservoir due to severe fibrosis from his radiation, and the case was terminated after repair of his vascular injuries.

After noting high rates of removal and replacements cases in both cohorts, we performed post-hoc subgroup analyses comparing virgin $(n=410)$ to removal and replacement cases $(\mathrm{n}=124$, Table S1). Removal and replacement cases did not have significantly higher total complication $(12.1 \%$ vs. $9.8 \%$, $\mathrm{P}=0.075)$ or device infection rates $(4.8 \%$ vs. $3.7 \%, \mathrm{P}=0.598)$. When stratifying complication rates between CI cohort by removal and replacement status there was a higher rate of malfunction in CI removal and replacements, 3 (13\%) vs. 2 (2\%), but no other differences between groups, however the sub-cohorts are likely too underpowered to reflect a relationship (Table S2).

\section{Discussion}

Inflatable penile prostheses are frequently placed in men with post-prostatectomy erectile dysfunction. In robotic assisted laparoscopic prostatectomy (RALP), the space of Retzius has been violated, putting patients at a higher risk for complications with traditional reservoir placement (21). Release of the bladder during transperitoneal RALP effectively "peritonealizes" the bladder, so future attempts to blindly place a reservoir deep to the transversalis fascia carries a higher risk of intraperitoneal placement, bowel, bladder, or vascular injury $(5,15)$. As such, there has been a recent movement to explore alternative reservoir placements that are now possible due to advances in lockout valve technology that prevent prior issues with auto- 
inflation $(9,22,23)$. These new approaches are a proven and safe alternative for men with a history of pelvic surgery undergoing IPP placement (17). Even after correcting for confounders including removal and replacement in our multivariable logistic analyses, use of a CI for alternative reservoir placement in our cohort did not predict total complications or device infections.

HSM positioning has been identified as an alternative approach in order to reduce risk of intraperitoneal placement (6). Despite common use of this technique, blind placement of a reservoir in the HSM space has potential for erroneous positioning. A cadaver study of $20 \mathrm{HSM}$ reservoir placements showed only $35 \%$ were in the intended HSM position, $45 \%$ were between the external and internal oblique fascia, $10 \%$ retroperitoneal, $5 \%$ preperitoneal, and $5 \%$ intraperitoneal (16). As a direct-vision reservoir insertion method, use of a counter-incision leads to more precise submuscular placement of the IPP reservoir and thus improved positioning.

Data from a recent resident cadaver simulation lab revealed high levels of anxiety regarding reservoir placement, with $42 \%$ of participants stating they did not feel confident with space of Retzius or submuscular reservoir placement (18). While the trainee population may not be generalizable to all practicing urologists, these were $1-2$ years from practice and therefore it is not unreasonable to assume representative of many young urologists. As most penile prostheses are not placed by high-volume implanters, use of a CI is an easy yet important option for alternative reservoir location placement by occasional implanters. This technique allows patients with prior pelvic surgeries access to a three-piece IPP, when previously they may have only been offered a malleable prosthesis or two-piece inflatable due to concerns over reservoir complication.

A single arm retrospective case series described the use of a CI for alternative reservoir placement into the lateral retroperitoneum in 80 patients who underwent IPP placement after radical cystectomy. All used an infrapubic rather than penoscrotal approach, and instead of submuscular placement the reservoir was positioned deep to the external oblique muscle using a CI $2 \mathrm{~cm}$ inferomedial to the anterior-superior iliac spine. At 5 years follow up, they reported 4 post-operative infections $(5.0 \%)$ and 5 revision surgeries $(6.25 \%)$, raising concern about the risk profile of an additional incision (24). However, a small case control study of 31 patients undergoing IPP after radical prostatectomy (94\%) or cystectomy used a similar surgical technique for retroperitoneal placement and reported no infections or malfunctions in their CI arm at 2 years (8). In contrast, in the control arm they reported $9.4 \%$ infection and $3 \%$ malfunction rates, but this did not reach statistical significance.

The use of a counter-incision in implant surgery raises concerns for infection due to an additional incision and a slightly longer operative time, which are traditionally thought to increase infection risk (25-28). Our study showed that though the median additional operative time increased by 17 minutes, the risk of device infection was similar between those surgeries with and without a CI. Further, it should be noted that the number of prior prostheses and rates of removal and replacement were significantly higher in the CI cohort, nearly $50 \%$, which carries a significantly higher risk of complications than a native insertion $(23,26)$. Even in this high-risk cohort of patients, infection rates among CI patients were only $2.0 \%$ and comparable to nonCI patients, demonstrating CIs are safe and feasible in patients with prior pelvic surgeries.

There were no herniations, bowel, or bladder injuries in the CI cohort as compared to two reservoir complications in the non-CI cohort. The vascular injury noted in the CI cohort was less likely due to the use of a CI and more likely secondary to the severe fibrosis caused by the patient's previous surgeries and radiation. The use of a CI, especially in a previously operated field, eliminated the possibility of an inadvertent visceral injury. Further, there is no dilation of the tract between reservoir and penoscrotal incision to place the reservoir, reducing the risk of a delayed reservoir herniation.

A high level of patient complexity was observed in this tertiary referral center population, as noted by high removal and replacement rates ( $23 \%$ of the overall cohort). These cases are inherently more challenging than primary placements, historically reflected in higher reported complication rates, including device infection (27). Removal and replacement cases comprised a higher fraction of the CI cohort, which could skew our results; however, we still did not observe higher complication or infection rates with CIs. In addition, on multivariate analysis, these more complex cases did not predict a significant increase in complications or infections. Given these multivariate results, we elected to include these more complex cases as they are reflective of our population, but acknowledge this may impact external validity.

A main limitation of this study is the selection bias given its retrospective nature. As previously stated, the CI cohort had significant differences from the non-CI cohort that 
certainly risk confounding our results. Some of these more complex patients had no alternative options other than a CI for reservoir placement, but it is worth noting that $28 \%$ of the non-CI cohort had a prior prostatectomy and $21 \%$ were removal and replacement cases, reflecting high patient complexity in both arms. In addition, due to the far smaller CI cohort it is possible our study is underpowered as complication rates, especially device infections, are thankfully rare. Being a retrospective database study, no power analyses were performed as we had no control over our sample size. As such, we plan to continue monitoring our outcomes as the cohorts become more robust. Furthermore, this retrospective database is not immune from potential underreporting or mis-classification bias, as we are dependent on the accuracy of the medical record. Finally, although we are limited by a single surgeon's data, we hope our data can be corroborated by other interested parties.

\section{Conclusions}

Overall, our experience using a CI for reservoir placement in this high-risk patient cohort demonstrates comparable safety and efficacy to standard technique and supports the consideration of CIs for alternative reservoir placements in prostate cancer survivors, who make up an overwhelming fraction of the erectile dysfunction population. For physicians not comfortable with alternative placement through a penoscrotal or infrapubic incision, this offers a safe alternative and permits use of a three-piece device in patients at risk for reservoir related complications.

\section{Acknowledgments}

Funding: None.

\section{Footnote}

Reporting Checklist: The authors have completed the STROBE reporting checklist. Available at http://dx.doi. org/10.21037/tau-20-923

Data Sharing Statement: Available at http://dx.doi. org/10.21037/tau-20-923

Peer Review File: Available at http://dx.doi.org/10.21037/ tau-20-923

Conflicts of Interest: All authors have completed the ICMJE uniform disclosure form (available at http://dx.doi. org/10.21037/tau-20-923). Dr. ACL reports personal fees from Coloplast Corporation, personal fees from Boston Scientific, outside the submitted work. The authors have no conflicts of interest to declare.

Ethical Statement: The authors are accountable for all aspects of the work in ensuring that questions related to the accuracy or integrity of any part of the work are appropriately investigated and resolved. The study was conducted in accordance with the Declaration of Helsinki (as revised in 2013). The study was approved by the institutional review board of Duke University (Pro00101391) and because of the retrospective nature of this research the requirement for informed consent was waived.

Open Access Statement: This is an Open Access article distributed in accordance with the Creative Commons Attribution-NonCommercial-NoDerivs 4.0 International License (CC BY-NC-ND 4.0), which permits the noncommercial replication and distribution of the article with the strict proviso that no changes or edits are made and the original work is properly cited (including links to both the formal publication through the relevant DOI and the license). See: https://creativecommons.org/licenses/by-nc-nd/4.0/.

\section{References}

1. Haglind E, Carlsson S, Stranne J, et al. Urinary incontinence and erectile dysfunction after robotic versus open radical prostatectomy: a prospective, controlled, nonrandomised trial. Eur Urol 2015;68:216-25.

2. Brusky J, Dikranin A, Aboseif S. Retrieval of inflatable penile prosthesis reservoir from the bladder. Int J Impot Res 2005;17:302-3.

3. Brison D, Ilbeigi P, Sadeghi-Nejad H. Reservoir repositioning and successful thrombectomy for deep venous thrombosis secondary to compression of pelvic veins by an inflatable penile prosthesis reservoir. J Sex Med 2007;4:1185-7.

4. Deho' F, Henry GD, Marone EM, et al. Severe vascular complication after implantation of a three-piece inflatable penile prosthesis. J Sex Med 2008;5:2956-9.

5. Morey AF, Cefalu CA, Hudak SJ. High submuscular placement of urologic prosthetic balloons and reservoirs via transscrotal approach. J Sex Med 2013;10:603-10.

6. Perito $\mathrm{P}, \mathrm{Wilson} \mathrm{S}$. The history of nontraditional or 
ectopic placement of reservoirs in prosthetic urology. Sex Med Rev 2016;4:190-3.

7. Henry G, Carrion R, Bella A, et al. 1858 Pertinent anatomical measurements of the retropubic space: a guide for inflatable penile prosthesis reservoir placement shows that the external iliac viein is much closer than thought. J Urol 2012;187:e751.

8. Hartman RJ Jr, Helfand BT, McVary KT. Outcomes of lateral retroperitoneal reservoir placement of threepiece penile prosthesis in patients following radical prostatectomy. Int J Impot Res 2010;22:279-83.

9. Clavell-Hernández J, Shah A, Wang R. Non-infectious reservoir-related complications during and after penile prosthesis placement. Sex Med Rev 2019;7:521-9.

10. Perito PE. Ectopic reservoir placement--no longer in the space of Retzius. J Sex Med 2011;8:2395-8.

11. Perito PE, Wilson SK. Traditional (retroperitoneal) and abdominal wall (ectopic) reservoir placement. J Sex Med 2011;8:656-9.

12. Karpman E, Sadeghi-Nejad H, Henry G, et al. Current opinions on alternative reservoir placement for inflatable penile prosthesis among members of the Sexual Medicine Society of North America. J Sex Med 2013;10:2115-20.

13. Chung PH, Morey AF, Tausch TJ, et al. High submuscular placement of urologic prosthetic balloons and reservoirs: 2 -year experience and patient-reported outcomes. Urology 2014;84:1535-40.

14. Stember DS, Kohler TS, Morey AF. Management of perforation injuries during and following penile prosthesis surgery. J Sex Med 2015;12 Suppl 7:456-61.

15. Hudak SJ. Reservoir placement considerations during inflatable penile prosthesis surgery. Curr Urol Rep 2019;20:8.

16. Ziegelmann MJ, Viers BR, Lomas DJ, et al. Ectopic penile prosthesis reservoir placement: an anatomic cadaver model of the high submuscular technique. J Sex Med 2016;13:1425-31.

17. Stember DS, Garber BB, Perito PE. Outcomes of

Cite this article as: Grimberg D, Wang S, Carlos E, Nosé B, Harper S, Lentz AC. Counter incision is a safe and effective method for alternative reservoir placement during inflatable penile prosthesis surgery. Transl Androl Urol 2020;9(6):2688-2696. doi: $10.21037 /$ tau-20-923 abdominal wall reservoir placement in inflatable penile prosthesis implantation: a safe and efficacious alternative to the space of Retzius. J Sex Med 2014;11:605-12.

18. Lentz AC, Rodríguez D, Davis LG, et al. Simulation training in penile implant surgery: assessment of surgical confidence and knowledge with cadaveric laboratory training. Sex Med 2018;6:332-8.

19. Eid JF. Penile implant: review of a "no-touch" technique. Sex Med Rev 2016;4:294-300.

20. Henry GD, Wilson SK, Delk JR 2nd, et al. Revision washout decreases penile prosthesis infection in revision surgery: a multicenter study. J Urol 2005;173:89-92.

21. Pearce SM, Pariser JJ, Karrison T, et al. Comparison of perioperative and early oncologic outcomes between open and robotic assisted laparoscopic prostatectomy in a contemporary population based cohort. J Urol 2016;196:76-81.

22. Hollenbeck BK, Miller DC, Ohl DA. The utility of lockout valve reservoirs in preventing autoinflation in penile prostheses. Int Urol Nephrol 2002;34:379-83.

23. Levine LA, Hoeh MP. Review of penile prosthetic reservoir: complications and presentation of a modified reservoir placement technique. J Sex Med 2012;9:2759-69.

24. Loh-Doyle J, Patil MB, Sawkar H, et al. 3-piece inflatable penile prosthesis placement following radical cystoprostatectomy and urinary diversion: technique and outcomes. J Sex Med 2018;15:907-13.

25. Mulcahy JJ. Current approach to the treatment of penile implant infections. Ther Adv Urol 2010;2:69-75.

26. Mulcahy JJ. The prevention and management of noninfectious complications of penile implants. Sex Med Rev 2015;3:203-13.

27. Pineda M, Burnett AL. Penile prosthesis infections-a review of risk factors, prevention, and treatment. Sex Med Rev 2016;4:389-98.

28. Krzastek SC, Smith R. An update on the best approaches to prevent complications in penile prosthesis recipients. Ther Adv Urol 2019;11:1756287218818076. 
Supplementary

Table S1 Complication rates stratified by removal and replacement status

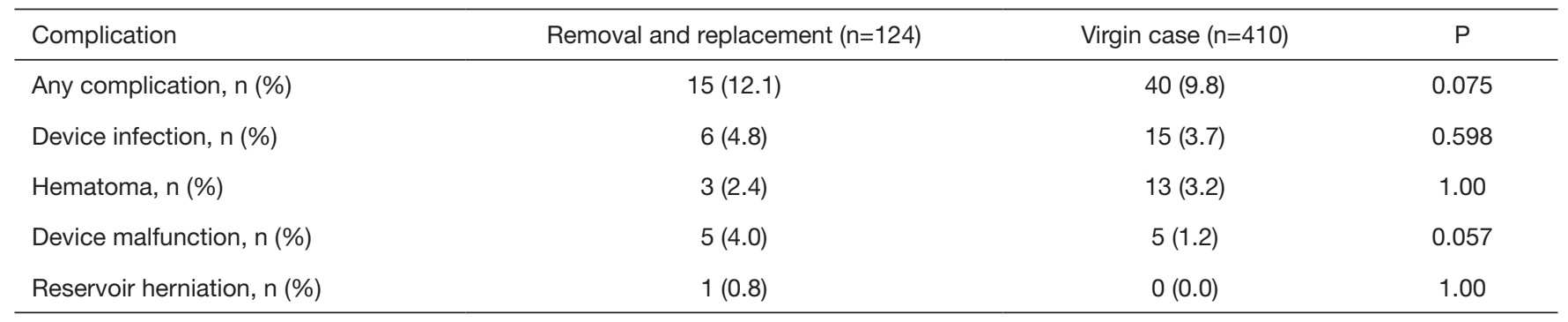

Table S2 Complication rates for virgin cases and removal and replacement cases stratified by CI

\begin{tabular}{|c|c|c|c|c|c|c|}
\hline Complication & \multicolumn{3}{|c|}{ Virgin cases $(n=410)$} & \multicolumn{3}{|c|}{ Removal and replacement cases $(n=124)$} \\
\hline Any complication, n (\%) & $5(17.9)$ & $35(9.2)$ & 0.243 & $2(8.7)$ & $13(12.9)$ & 0.841 \\
\hline Device infection, n (\%) & $1(3.6)$ & $14(3.7)$ & 1.00 & $0(0.0)$ & $6(5.9)$ & 0.592 \\
\hline Hematoma, n (\%) & $1(3.6)$ & $12(3.1)$ & 0.607 & $2(8.7)$ & $1(1.0)$ & 0.088 \\
\hline Reservoir herniation, n (\%) & $0(0.0)$ & $0(0.0)$ & 1.00 & $0(0.0)$ & $1(1.0)$ & 1.00 \\
\hline
\end{tabular}

$\mathrm{Cl}$, counter incision. 